\title{
THE DYNAMIC PROGRAMMING EQUATION FOR SECOND ORDER STOCHASTIC TARGET PROBLEMS*
}

\author{
H. METE SONER ${ }^{\dagger}$ AND NIZAR TOUZI ${ }^{\ddagger}$
}

\begin{abstract}
Motivated by applications in mathematical finance [U. Cetin, H. M. Soner, and N. Touzi, "Options hedging for small investors under liquidity costs," Finance Stoch., to appear] we continue our study of second order backward stochastic equations. In this paper, we derive the dynamic programming equation for a certain class of problems which we call the second order stochastic target problems. In contrast with previous formulations of similar problems, we restrict control processes to be continuous. This new framework enables us to apply our results to a larger class of models. Also the resulting derivation is more transparent. The main technical tool is the geometric dynamic programming principle in this context, and it is proved by using the framework developed in [H. M. Soner and N. Touzi, J. Eur. Math. Soc. (JEMS), 8 (2002), pp. 201-236].
\end{abstract}

Key words. stochastic target problem, gamma process, geometric dynamic programming, viscosity solutions

AMS subject classifications. 91B28, 35K55, 60H30

DOI. $10.1137 / 07071130 \mathrm{X}$

1. Introduction. The stochastic target problems were introduced in [17] as a natural extension of the superhedging problem in financial mathematics. In this initial study, only target sets which are epigraphs were considered. General target sets were then studied by the authors in [18] and stochastic representations for geometric flows were derived. In particular, some front propagation problems and extensions of the classical mean curvature flow were studied in [18]. Bouchard [2] and then Saintier [15] extended these results to a more general class of processes, including Levy processes.

Stochastic target problems are also closely connected to the theory of backward stochastic differential equations (BSDEs) initiated in $[13,14]$. The theory of BSDEs has applications in many diverse fields, especially in mathematical finance [8]. In particular, recently BSDEs have been used to obtain stochastic representations for the solutions of quasi-linear PDEs. This connection also provides novel probabilistic numerical methods for these equations. In [6], a class of second order BSDEs (2BSDEs) is introduced to extend this stochastic representation to the class of fully nonlinear PDEs. In this connection second order stochastic target problems were also introduced and used critically in the uniqueness result for 2BSDEs.

This paper continues the study of second order stochastic target problems. In [6] the minimal value is proved to be a viscosity supersolution of the corresponding dynamic programming equation. The main results of this paper, Theorems 3.1 and 3.2 , are similar. Indeed, Theorem 3.2 states that the value function of the second order

* Received by the editors December 19, 2007; accepted for publication (in revised form) April 13, 2009; published electronically July 17, 2009. This research is part of the Chair Financial Risks of the Risk Foundation sponsored by Société Générale, the Chair Derivatives of the Future sponsored by the Fédération Bancaire Française, and the Chair Finance and Sustainable Development sponsored by EDF and Calyon.

http://www.siam.org/journals/sicon/48-4/71130.html

†Sabanci University, Istanbul, Turkey (msoner@sabanciuniv.edu). The work of this author was partly supported by the Turkish Technical and Scientific Council, TÜBITAK, and the European Research Council under grant 228053-FiRM.

${ }^{\ddagger}$ CMAP, Ecole Polytechnique, 91128 Palaiseau cedex, Paris, France (nizar.touzi@polytechnique. edu). 
stochastic target problem is a viscosity solution of the dynamic programming equation. This result is analogous to the classical Perron's method in harmonic analysis and in viscosity solutions which states that the minimal supersolution is a solution.

The authors studied a closely related problem in [4]. However, in contrast to [4], in this paper the control processes are continuous. Precise formulation of the control space is given in section 2. Technically, this restriction to continuous control processes implies a more involved proof of the dynamic programming principle, as reported in this paper. However, it induces a considerable simplification of the derivation of the dynamic programming equation.

This paper is motivated by a pricing problem in an illiquid market [3] and the earlier work of the authors on gamma constraints [16, 17]. Indeed, in the related work of the authors with Cetin [3], the continuity of the control processes is crucial.

The paper is organized as follows. The second order stochastic target problem is formulated in section 2. The main results are collected in section 3. Sections 4 and 5 are dedicated, respectively, to the derivation of the viscosity property of the value function and the proof of the geometric dynamic programming principle. Finally, in section 6 we prove the properties of the value function at final time.

Notation. We use the following notation throughout the paper.

- $d \geq 1$ is an integer denoting the dimension.

- $\mathcal{M}^{d}$ is the set of all $d \times d$ matrices with real components.

- $B^{\prime}$ is the transpose of a matrix $B \in \mathcal{M}^{d}$ and $\operatorname{Tr}[B]$ its trace.

- $\mathcal{M}_{\text {inv }}^{d}$ is the set of all invertible matrices in $\mathcal{M}^{d}$.

- $\mathcal{S}^{d}$ is the set of all symmetric matrices in $\mathcal{M}^{d}$.

- $\mathcal{S}_{+}^{d}$ are all positive semidefinite matrices in $\mathcal{M}^{d}$.

- For $x \in \mathbb{R}^{d},|x|:=\left(x_{1}^{2}+\cdots+x_{d}^{2}\right)^{1 / 2}$.

- $B \in \mathcal{M}^{d},|B|:=\left(\sum_{i, j=1}^{d} B_{i j}^{2}\right)^{1 / 2}$.

Equalities and inequalities between random variables are always understood in the almost sure sense.

\section{Problem formulation.}

2.1. Uncontrolled state variable. Throughout this paper, we fix a finite time horizon $T \in(0, \infty)$, and we consider a $d$-dimensional Brownian motion $\left\{W_{t}\right\}_{t \in[0, T]}$ on a complete probability space $(\Omega, \mathcal{F}, P)$. For $t \in[0, T]$, we denote by $\mathbb{F}=\left(\mathcal{F}_{t}\right)_{t \in[0, T]}$ the augmented filtration generated by $\left\{W_{t}\right\}_{t \in[0, T]}$.

Let $\mu: \mathbb{R}^{d} \rightarrow \mathbb{R}^{d}$ and $\sigma: \mathbb{R}^{d} \rightarrow \mathcal{M}_{\text {inv }}^{d}$ be two functions satisfying the standard Lipschitz and growth conditions for all $x, y \in \mathbb{R}^{d}$,

$$
|\mu(x)-\mu(y)|+|\sigma(x)-\sigma(y)| \leq K|x-y|, \quad|\mu(x)|+|\sigma(x)| \leq K(1+|x|),
$$

for some constant $K$. Then, for every initial condition $(s, x) \in[0, T] \times \mathbb{R}^{d}$, the stochastic differential equation

$$
X_{t}=x+\int_{s}^{t}\left[\mu\left(X_{u}\right) d u+\sigma\left(X_{u}\right) d W_{u}\right], \quad t \in[s, T]
$$

has a unique strong solution $\left\{X_{t}^{s, x}\right\}_{t \in[s, T]}$; see, for instance, Theorem 5.2.9 in Karatzas and Shreve [11]. To introduce our notation, we also recall the classical Itô's formula which holds for all $\varphi \in C^{1,2}\left([0, T] \times \mathbb{R}^{d}\right)$ and $t \in[0, T]$ :

$$
\varphi\left(t, X_{t}^{s, x}\right)=\varphi(s, x)+\int_{s}^{t} \mathcal{L} \varphi\left(r, X_{r}^{s, x}\right) d r+\int_{s}^{t} D \varphi\left(r, X_{r}^{s, x}\right)^{\prime} d X_{r}^{s, x}
$$


where

$$
\mathcal{L} \varphi(t, x)=\frac{\partial \varphi}{\partial t}(t, x)+\frac{1}{2} \operatorname{Tr}\left[D^{2} \varphi(t, x) \sigma(x) \sigma(x)^{\prime}\right],
$$

and $D \varphi, D^{2} \varphi$ are the partial gradient and Hessian matrix of $\varphi$ with respect to the $x$-variables.

2.2. Controlled state variable. This class of controls is very similar to the one introduced in [6]. The main difference is the new relaxed restriction (2.2), below, placed on the drift process $A$ and on the modulus of continuity of $\Gamma$. In [6] an $L^{\infty}$ bound was placed on the drift $A_{t}$, and $\Gamma$ was assumed to be Lipschitz.

To state the control space, first we need to define a norm-like function. For $B, b \geq 0$ and $(s, x) \in[0, T] \times \mathbb{R}^{d}$, we define the norm of an $\mathbb{F}$-progressively measurable process $\left\{H_{t}\right\}_{t \in[s, T]}$ by

$$
\|H\|_{s, x}^{B, b}:=\left\|\sup _{s \leq t \leq T} \frac{\left|H_{t}\right|}{1+\left|X_{t}^{s, x}\right|^{B}}\right\|_{L^{b}(\Omega, P)} .
$$

Fix $B \geq 0, b \in(0,1)$. For all $(s, x) \in[0, T] \times \mathbb{R}^{d}$ and $m>0$, let $\mathcal{A}_{m, b}^{s, x}$ be the class of all (control) processes of the form

$$
\begin{aligned}
& Z_{t}=z+\int_{s}^{t} A_{r} d r+\int_{s}^{t} \Gamma_{r} d X_{r}^{s, x}, \quad t \in[s, T], \\
& \Gamma_{t}=\gamma+\int_{s}^{t} a_{r} d r+\int_{s}^{t} \xi_{r} d X_{r}^{s, x}, \quad t \in[s, T],
\end{aligned}
$$

where $\left\{Z_{t}\right\}_{t \in[s, T]}$ is $\mathbb{R}^{d}$-valued and $\left\{\Gamma_{t}\right\}_{t \in[s, T]}$ is $\mathcal{S}^{d}$-valued. Notice that both $Z$ and $\Gamma$ are continuous functions of time. Further, all the above processes are assumed to be $\mathbb{F}$-progressively measurable and satisfy the following inequalities:

$$
\begin{gathered}
\|Z\|_{s, x}^{B, \infty} \leq m, \quad\|\Gamma\|_{s, x}^{B, \infty} \leq m, \quad\|\xi\|_{s, x}^{B, 2} \leq m, \\
\|A\|_{s, x}^{B, b} \leq m, \quad\|a\|_{s, x}^{B, b} \leq m .
\end{gathered}
$$

Set $\mathcal{A}^{s, x}:=\bigcup_{b \in(0,1]} \bigcup_{m \geq 0} \mathcal{A}_{m, b}^{s, x}$. It is clear that $\mathcal{A}^{s, x}:=\bigcup_{m \geq 0} \mathcal{A}_{m}^{s, x}$, where $\mathcal{A}_{m}^{s, x}:=$ $\mathcal{A}_{m,(1 / m)}^{s, x}$

Notice that these classes of controls also depend on the parameter $B$, but this dependence is suppressed. We will always fix $B \geq 1$ larger than any exponent that will appear in our assumptions.

Remark 2.1. Any element $Z \in \mathcal{A}^{s, x}$ may be identified by the initial data $(z, \gamma)$ and the processes $A, a, \xi$. When we allow the controller to choose an element from $\mathcal{A}^{s, x}$, we implicitly allow her to determine the initial datum as well as the processes. However, in section 5 below, we will allow the controller to choose only the process but not the initial datum. So for future reference, we define the set $\mathcal{A}^{s, x, z, \gamma}$ to be the set of all processes $Z$ and $\Gamma$ as above with fixed initial data $Z_{s}=z$ and $\Gamma_{s}=\gamma$. The control set $\mathcal{A}_{m}^{s, x, z, \gamma}$ is defined analogously. Then it is clear that $\mathcal{A}^{s, x, z, \gamma}:=\bigcup_{m \geq 0} \mathcal{A}_{m}^{s, x, z, \gamma}$.

Remark 2.2. The above control processes are defined through the restrictions (2.1) and (2.2). First condition (2.1) is analogous to the growth conditions used in the PDE literature and seems to be quite general. However, the structure of the second 
restriction (2.2) is technically very important. First, without any assumption of this type, the separation between the processes $A, \Gamma$ and $a, \xi$ is not clear. Moreover, the uniform approximation results of Leventhal and Skorohod [12] and Bank and Baum [1] apply to our problem, rendering the $\Gamma$ process irrelevant. For this reason, in our previous studies, we always placed this type of a restriction on the portfolio process. Condition (2.2) used in this paper is the weakest restriction employed in these studies. Indeed, the minimal assumptions on $a$ and $\xi$ are such that the conclusions of Lemma 4.3 below still hold and (2.2) is very close to this minimal assumption. We chose not to state this technical minimal assumption and used (2.2) for simplicity. We next consider a continuous function $f:[0, T) \times \mathbb{R}^{d} \times \mathbb{R} \times \mathbb{R}^{d} \times \mathcal{S}^{d} \rightarrow \mathbb{R}$ satisfying the following Lipschitz and growth assumptions.

(A1) For every $N \geq 1$ there exists a constant $F_{N}$ such that

$$
|f(t, x, y, z, \gamma)-f(t, x, \tilde{y}, z, \gamma)| \leq F_{N}|y-\tilde{y}|
$$

for all $(t, x, y, z, \gamma) \in[0, T] \times \mathbb{R}^{d} \times \mathbb{R} \times \mathbb{R}^{d} \times \mathcal{S}^{d}, \tilde{y} \in \mathbb{R}$ satisfying

$$
\max \{|x|,|y|,|\tilde{y}|,|z|,|\gamma|\} \leq N .
$$

(A2) There exist constants $F$ and $p \geq 0$ such that

$$
|f(t, x, y, z, \gamma)| \leq F\left(1+|x|^{p}+|y|+|z|^{p}+|\gamma|^{p}\right)
$$

for all $(t, x, y, z, \gamma) \in[0, T] \times \mathbb{R}^{d} \times \mathbb{R} \times \mathbb{R}^{d} \times \mathcal{S}^{d}$.

Now consider the stochastic differential equation

$$
d Y_{t}=f\left(t, X_{t}^{s, x}, Y_{t}, Z_{t}, \Gamma_{t}\right) d t+Z_{t} \circ d X_{t}^{s, x}, \quad t \in[s, T),
$$

with initial data $Y_{s}=y$. Here $\circ$ denotes the Fisk-Stratonovich integral. Due to the form of the $Z$ process, this integral can be expressed in terms of standard Itô integral,

$$
Z_{t} \circ d X_{t}^{s, x}=Z_{t} \cdot d X_{t}^{s, x}+\frac{1}{2} \operatorname{Tr}\left[\sigma^{t} \sigma \Gamma_{t}\right] d t .
$$

Under the above assumptions (A1), (A2) and (2.1) on $Z$, it follows that for all $y \in \mathbb{R}$ and $Z \in \mathcal{A}^{s, x}$, this equation has a unique strong solution $\left\{Y_{t}^{s, x, y, Z}\right\}_{t \in[s, T]}$. This can be shown, for instance, with the arguments in the proofs of Theorems 2.3, 2.4, and 3.1 in Chapter IV of Ikeda and Watanabe [10].

We also assume the following control on the monotonicity condition.

(A3) There exists a constant $c_{0}>0$ such that

$$
\begin{aligned}
& f\left(t, x, y^{\prime}, z, \gamma\right)-f(t, x, y, z, \gamma) \geq-c_{0}\left(y^{\prime}-y\right) \text { for every } y^{\prime} \geq y, \\
& \text { and }(t, x, z, \gamma) \in[0, T) \times \mathbb{R}^{d} \times \mathbb{R}^{d} \times \mathcal{S}^{d} .
\end{aligned}
$$

2.3. The second order stochastic target problem. Let $g: \mathbb{R}^{d} \rightarrow \mathbb{R}$ be a continuous function satisfying the following growth condition.

(A4) Terminal condition $g$ is continuous and there exist constants $G$ and $p$ such that

$$
|g(x)| \leq G\left(1+|x|^{p}\right) \quad \forall x \in \mathbb{R}^{d} .
$$


We are now in a position to define the key object of our study. For $(s, x) \in[0, T] \times \mathbb{R}^{d}$,

$$
V(s, x):=\inf \left\{y \in \mathbb{R} \mid \exists Z \in \mathcal{A}^{s, x} \text { so that } Y_{T}^{s, x, y, Z} \geq g\left(X_{T}^{s, x}\right) \text { a.s. }\right\} .
$$

For the benefit of the reader, we recall the dynamics of all the processes used in the above definition. Also recall that the class of admissible processes $\mathcal{A}^{s, x}$ is given in the previous subsection. We use the short notation $X:=X^{s, x}, Y:=Y^{s, x, y, Z}$. Then, for all $t \in[s, T]$,

$$
\begin{aligned}
X_{t} & =x+\int_{s}^{t} \mu\left(X_{r}\right) d r+\int_{s}^{t} \sigma\left(X_{r}\right) d W_{r} \\
Y_{t} & =y+\int_{s}^{t} f\left(t, X_{r}, Y_{r}, Z_{r}, \Gamma_{r}\right) d r+\int_{s}^{t} Z_{r} \circ d X_{r} \\
& =y+\int_{s}^{t} f\left(t, X_{r}, Y_{r}, Z_{r}, \Gamma_{r}\right) d r+\int_{s}^{t}\left[Z_{t} \cdot d X_{t}+\frac{1}{2} \operatorname{Tr}\left[\sigma^{t} \sigma \Gamma_{t}\right] d t\right], \\
Z_{t} & =z+\int_{s}^{t} A_{r} d r+\int_{s}^{t} \Gamma_{r} d X_{r}, \\
\Gamma_{t} & =\gamma+\int_{s}^{t} a_{r} d r+\int_{s}^{t} \xi_{r} d X_{r} .
\end{aligned}
$$

The main objective of our study is to derive a dynamic programming equation for this problem. As it is classical, we will use the theory of viscosity solutions for this derivation. For this theory, we refer the reader to the survey article of Crandall, Ishii, and Lions [7] and to the book of Fleming and Soner [9].

Notice that this problem does not fit into the class of stochastic target problems studied by Soner and Touzi [17], as the dynamics of the controlled process $Y$ are affected by the process $\Gamma$ in the spirit of [6]. For this reason, we shall refer to the above control problem as a second order stochastic target problem.

Under the standing assumptions (A1)-(A4), it follows from Proposition 4.5 in [6] that the value function $V$ is bounded from below. To ensure that $V$ is finite we need the following assumption.

(A5) For each $(t, x)$, there exists a portfolio $Z \in \mathcal{A}^{t, x}$ and an initial data $y$ so that $Y_{T}^{t, x, y, Z} \geq g\left(X_{T}^{t, x}\right)$ a.s.

Under the above assumption, $V<\infty$. Then, by a minor modification of the proof of Theorem 4.2 in [6], we show in section 4 that the value function is a viscosity supersolution of the corresponding dynamic programming equation

$$
-\frac{\partial}{\partial t} v(t, x)+\hat{f}\left(t, x, v(t, x), D v(t, x), D^{2} v(t, x)\right)=0
$$

on $[0, T) \times \mathbb{R}^{d}$, where

$$
\hat{f}(t, x, y, z, \gamma):=\sup _{\beta \in \mathcal{S}_{+}^{d}} f(t, x, y, z, \gamma+\beta) .
$$

The function $\hat{f}$ is the smallest majorant of $f$ which is nonincreasing in the $\gamma$ argument and is called the parabolic envelope of $f$; see [5].

In section 4 , we also prove that the value function is a viscosity subsolution, and thus a solution of the above equation. 
In our previous papers $[4,5]$, the viscosity subsolution property was proved after enlarging the set of control processes $\mathcal{A}^{s, x}$ and also by allowing for jumps in the $Z$ process. This control relaxation was used for the formulation of the geometric dynamic programming principle, which is the main ingredient for the derivation of the dynamic programming equation. However, the inclusion of the jumps made the proof of the viscosity supersolution property technical. Moreover, the problems studied in these papers consider only specific functions $f$ related to certain pricing problems.

The main technical contribution of this paper is to prove that the value function $V$ is a viscosity solution of the dynamic programming equation (2.4) without any control relaxation and any jump components.

3. Main results. Assumptions (A1)-(A5) are assumed to hold throughout the paper.

This section collects the statements of the main results of this paper. We first state the geometric dynamic programming principle in the context of the second order stochastic target problem (2.3). Notice that the following result is not included in previous studies as we restrict the control processes to be continuous.

Theorem 3.1 (geometric dynamic programming principle). For any $(s, x) \in$ $[0, T) \times \mathbb{R}^{d}$, and a stopping time $\theta \in[s, T]$,

$$
V(s, x)=\inf \left\{y \in \mathbb{R} \mid \exists Z \in \mathcal{A}^{s, x} \text { so that } Y_{\theta}^{s, x, y, Z} \geq V\left(\theta, X_{\theta}^{s, x}\right) \text { a.s. }\right\} .
$$

Then the dynamic programming equation (2.4) is the infinitesimal analogue of the above geometric dynamic programming principle. Equation (2.4) is obtained in two steps. The supersolution property is deduced from the following consequence of (3.1) in subsection 4.2 .

(GDP1) For every $\varepsilon>0$, there exist $y_{\varepsilon} \in[V(s, x), V(s, x)+\varepsilon]$ and $Z_{\varepsilon} \in \mathcal{A}^{s, x}$ such that

$$
Y_{\theta}^{s, x, y_{\varepsilon}, Z_{\varepsilon}} \geq V\left(\theta, X_{\theta}^{s, x}\right)
$$

In subsection 4.1, the subsolution property is proved using the following claim, again implied by (3.1).

(GDP2) For every $y<V(s, x)$ and every $Z \in \mathcal{A}^{s, x}$,

$$
\mathbb{P}\left[Y_{\theta}^{s, x, y, Z} \geq V\left(\theta, X_{\theta}^{s, x}\right)\right]<1 .
$$

Notice that (3.1) is equivalent to (GDP1)-(GDP2).

Next, we introduce the semicontinuous envelopes.

$$
V_{*}(t, x):=\liminf _{\left(t^{\prime}, x^{\prime}\right) \rightarrow(t, x)} V\left(t^{\prime}, x^{\prime}\right) \quad \text { and } \quad V^{*}(t, x):=\limsup _{\left(t^{\prime}, x^{\prime}\right) \rightarrow(t, x)} V\left(t^{\prime}, x^{\prime}\right)
$$

for $(t, x) \in[0, T] \times \mathbb{R}^{d}$.

THEOREM 3.2. The value function $V$ is finite and is a viscosity solution of the dynamic programming equation $(2.4)$ on $[0, T] \times \mathbb{R}^{d}$; i.e., $V_{*}$ and $V^{*}$ are, respectively, the viscosity supersolution and subsolution of (2.4).

Our final result is on the behavior of the value function at the final time $T$. Under our assumptions, we will show that $V_{*}(T, x) \geq g(x)$. To prove the reverse inequality, we need an assumption stronger than (A5). Let $\hat{f}$ be as in (2.5). 
(A5)' For every $\varepsilon \in(0,1]$ and $x_{0} \in \mathbb{R}^{d}$, there exist $t\left(\varepsilon, x_{0}\right)<T$ and a smooth supersolution $U^{\varepsilon, x_{0}} \in C\left(\left[t\left(\varepsilon, x_{0}\right), T\right] \times \mathbb{R}^{d}\right)$ of (2.4) with $f=\hat{f}$ on $\left[t\left(\varepsilon, x_{0}\right), T\right] \times \mathbb{R}^{d}$ and satisfying

$$
\lim _{\varepsilon \downarrow 0} U^{\varepsilon, x_{0}}\left(T, x_{0}\right)=g\left(x_{0}\right), \quad U^{\varepsilon}(T, x) \geq g(x) \quad \forall x \in \mathbb{R}^{d} .
$$

In section 6 , we derive several conditions on the functions $g, f$ that are sufficient for (A5)'.

ThEOREm 3.3. Assume (A5)'. Then the value function $V$ satisfies the terminal condition $V(T, x)=g$, i.e.,

$$
V_{*}(T, x) \geq g(x), \quad V^{*}(T, x) \leq g(x) \quad \forall x \in \mathbb{R}^{d} .
$$

Clearly the above viscosity properties need to be complemented by a comparison result in order to provide a characterization of the second order stochastic control problem. Indeed, several deep comparison results are available in the theory of viscosity solutions (see, for instance, [7]). Moreover, this issue is discussed in detail in [6]. We refer the reader to these articles for this very important point. Such a comparison result is in fact an implicit assumption on the nonlinearity $f$.

4. Proof of Theorem 3.2. In this section, we prove the sub- and supersolution properties separately in the following two subsections. Our proof assumes the geometric dynamic programming principle (3.1). The proof of (3.1) will be given in section 5 .

4.1. The viscosity subsolution property. In this subsection, we prove that $V^{*}$ is a subsolution of the dynamic programming equation (2.4).

LEMMA 4.1. $V^{*}$ is a viscosity subsolution of (2.4).

Proof. Set

$$
\mathbf{Q}:=[0, T) \times \mathbb{R}^{d} .
$$

Let $\left(t_{0}, x_{0}\right) \in \mathbf{Q}$ and $\varphi \in C^{\infty}(\mathbf{Q})$ be such that

$$
0=\left(V^{*}-\varphi\right)\left(t_{0}, x_{0}\right)>\left(V^{*}-\varphi\right)(t, x) \text { for } \mathbf{Q} \ni(t, x) \neq\left(t_{0}, x_{0}\right) .
$$

In order to show that $V^{*}$ is a subsolution of (2.4), we assume the contrary, i.e., suppose that there is $\beta \in \mathcal{S}_{+}^{d}$ satisfying

$$
-\frac{\partial \varphi}{\partial t}\left(t_{0}, x_{0}\right)+f\left(t_{0}, x_{0}, \varphi\left(t_{0}, x_{0}\right), D \varphi\left(t_{0}, x_{0}\right), D^{2} \varphi\left(t_{0}, x_{0}\right)+\beta\right)>0 .
$$

We will then prove the subsolution property by contradicting (GDP2).

Step 1. Set

$$
\begin{aligned}
\psi(t, x) & :=\varphi(t, x)+\beta\left(x-x_{0}\right) \cdot\left(x-x_{0}\right) \\
h(t, x) & :=-\frac{\partial \psi}{\partial t}(t, x)+f\left(t, x, \psi(t, x), D \psi(t, x), D^{2} \psi(t, x)\right) .
\end{aligned}
$$

In view of $(4.2), h\left(t_{0}, x_{0}\right)>0$. Since the nonlinearity $f$ is continuous and $\varphi$ is smooth, the subset

$$
\mathcal{N}:=\left\{(t, x) \in \mathbf{Q} \cap B_{1}\left(t_{0}, x_{0}\right): h(t, x)>0\right\}
$$


is an open bounded neighborhood of $\left(t_{0}, x_{0}\right)$. Here $B_{1}\left(t_{0}, x_{0}\right)$ is the unit ball of $\mathbf{Q}$ centered at $\left(t_{0}, x_{0}\right)$. Since $\left(t_{0}, x_{0}\right)$ is defined by (4.1) as the point of strict maximum of the difference $\left(V^{*}-\varphi\right)$, we conclude that

$$
-\eta:=\max _{\partial \mathcal{N}}\left(V^{*}-\varphi\right)<0 .
$$

Next we fix $\lambda \in(0,1)$, and choose $(\hat{t}, \hat{x})$ so that

$$
(\hat{t}, \hat{x}) \in \mathcal{N}, \quad\left|\hat{x}-x_{0}\right| \leq \lambda \eta, \quad \text { and } \quad|V(\hat{t}, \hat{x})-\varphi(\hat{t}, \hat{x})| \leq \lambda \eta
$$

Set $\hat{X}:=X^{\hat{t}, \hat{x}}$ and define a stopping time by

$$
\theta:=\inf \left\{t \geq \hat{t}:\left(t, \hat{X}_{t}\right) \notin \mathcal{N}\right\} .
$$

Then $\theta>\hat{t}$. The pathwise continuity of $\hat{X}$ implies that $\left(\theta, \hat{X}_{\theta}\right) \in \partial \mathcal{N}$. Then, by (4.3),

$$
V^{*}\left(\theta, \hat{X}_{\theta}\right) \leq \varphi\left(\theta, \hat{X}_{\theta}\right)-\eta \text {. }
$$

Step 2. Consider the control process

$$
\hat{z}:=D \psi(\hat{t}, \hat{x}), \hat{A}_{t}:=\mathcal{L} D \psi\left(t, \hat{X}_{t}\right) \mathbf{1}_{[\hat{t}, \theta)}(t), \quad \text { and } \quad \hat{\Gamma}_{t}:=D^{2} \psi\left(t, \hat{X}_{t}\right) \mathbf{1}_{[\hat{t}, \theta)}(t)
$$

so that, for $t \in[\hat{t}, \theta]$,

$$
\hat{Z}_{t}:=\hat{z}+\int_{\hat{t}}^{t} \hat{A}_{r} d r+\int_{\hat{t}}^{t} \hat{\Gamma}_{r} d \hat{X}_{r}=D \psi\left(t, \hat{X}_{t}\right) .
$$

Since $\mathcal{N}$ is bounded and $\varphi$ is smooth, we directly conclude that $\hat{Z} \in \mathcal{A}^{\hat{t}, \hat{x}}$.

Step 3. Set $\hat{y}<V(\hat{t}, \hat{x}), \hat{Y}_{t}:=Y_{t}^{\hat{t}, \hat{x}, \hat{y}, \hat{Z}}$, and $\hat{\Psi}_{t}:=\psi\left(t, \hat{X}_{t}\right)$. Clearly, the process $\Psi$ is bounded on $[\hat{t}, \theta]$. For later use, we need to show that the process $\hat{Y}$ is also bounded. By definition, $\hat{Y}_{\hat{t}}<\Psi_{\hat{t}}$. Consider the stopping times

$$
\tau_{0}:=\inf \left\{t \geq \hat{t}: \Psi_{t}=\hat{Y}_{t}\right\}
$$

and, with $N:=\eta^{-1}$,

$$
\tau_{\eta}:=\inf \left\{t \geq \hat{t}: \hat{Y}_{t}=\Psi_{t}-N\right\}
$$

We will show that for a sufficiently large $N$, both $\tau_{0}=\tau_{\eta}=\theta$. This proves that as $\Psi, \hat{Y}$ is also bounded on $[\hat{t}, \theta]$.

Set $\hat{\theta}:=\theta \wedge \tau_{0} \wedge \tau_{\eta}$. Since both processes $\hat{Y}$ and $\Psi$ solve the same stochastic differential equation, it follows from the definition of $\mathcal{N}$ that for $t \in[\hat{t}, \hat{\theta}]$

$$
\begin{aligned}
d\left(\Psi_{t}-\hat{Y}_{t}\right) & =\left[\frac{\partial \psi}{\partial t}\left(t, \hat{X}_{t}\right)-f\left(t, \hat{X}_{t}, \hat{Y}_{t}, \hat{Z}_{t}, \hat{\Gamma}_{t}\right)\right] d t \\
& \leq\left[f\left(t, \hat{X}_{t}, \Psi_{t}, \hat{Z}_{t}, \hat{\Gamma}_{t}\right)-f\left(t, \hat{X}_{t}, \hat{Y}_{t}, \hat{Z}_{t}, \hat{\Gamma}_{t}\right)\right] d t \\
& \leq F_{N}\left(\Psi_{t}-\hat{Y}_{t}\right) d t
\end{aligned}
$$


by the local Lipschitz property (A1) of $f$. Then

$$
0 \leq \Psi_{\hat{\theta}}-\hat{Y}_{\hat{\theta}} \leq\left(\Psi_{\hat{t}}-\hat{Y}_{\hat{t}}\right) e^{F_{N} T} \leq \frac{1}{2}\|\beta\| \lambda^{2} e^{F_{N} T} \eta^{2},
$$

where the last inequality follows from (4.4). This shows that, for $\lambda$ sufficiently small, $\hat{\theta}<\tau_{\eta}$, and therefore the difference $\Psi-\hat{Y}$ is bounded. Since $\Psi$ is bounded, this implies that $\hat{Y}$ is also bounded for small $\eta$.

Step 4 . In this step we will show that for any initial data

$$
\hat{y} \in[V(\hat{t}, \hat{x})-\lambda \eta, V(\hat{t}, \hat{x})),
$$

we have $\hat{Y}_{\theta} \geq V\left(\theta, X_{\theta}\right)$. This inequality is in contradiction to (GDP2) as $\hat{Y}_{\hat{t}}=\hat{y}<$ $V(\hat{t}, \hat{x})$. This contradiction proves the subsolution property.

Indeed, using $\hat{y} \geq V(\hat{t}, \hat{x})-\lambda \eta$ and $V \leq V^{*} \leq \varphi$ together with (4.3) and (4.4), we obtain the following sequence of inequalities:

$$
\begin{aligned}
\hat{Y}_{\theta}-V\left(\theta, \hat{X}_{\theta}\right) \geq & \hat{Y}_{\theta}-\varphi\left(\theta, \hat{X}_{\theta}\right)+\eta \\
= & {[\hat{y}-\varphi(\hat{t}, \hat{x})+\eta]+\int_{\hat{t}}^{\theta}\left[d \hat{Y}_{t}-d \varphi\left(t, \hat{X}_{t}\right)\right], } \\
\geq & \eta(1-2 \lambda)+\int_{\hat{t}}^{\theta}\left[f\left(t, \hat{X}_{t}, \hat{Y}_{t}, \hat{Z}_{t}, \hat{\Gamma}_{t}\right) d t+\hat{Z}_{t} \circ d \hat{X}_{t}-d \varphi\left(t, \hat{X}_{t}\right)\right] \\
\geq & \eta(1-2 \lambda)+\frac{1}{2} \beta\left(\hat{X}_{\theta}-\hat{x}\right) \cdot\left(\hat{X}_{\theta}-\hat{x}\right) \\
& \quad+\int_{\hat{t}}^{\theta}\left[f\left(t, \hat{X}_{t}, \hat{Y}_{t}, \hat{Z}_{t}, \hat{\Gamma}_{t}\right) d t+\hat{Z}_{t} \circ d \hat{X}_{t}-d \psi\left(t, \hat{X}_{t}\right)\right] \\
\geq & \eta(1-2 \lambda)+\int_{\hat{t}}^{\theta}\left[f\left(t, \hat{X}_{t}, \hat{Y}_{t}, \hat{Z}_{t}, \hat{\Gamma}_{t}\right) d t+\hat{Z}_{t} \circ d \hat{X}_{t}-d \psi\left(t, \hat{X}_{t}\right)\right],
\end{aligned}
$$

where the last inequality follows from the nonnegativity of the symmetric matrix $\beta$. We next use Itô's formula and the definition of $\mathcal{N}$ to arrive at

$$
\hat{Y}_{\theta}-V\left(\theta, \hat{X}_{\theta}\right) \geq \eta(1-2 \lambda)+\int_{\hat{t}}^{\theta}\left[f\left(t, \hat{X}_{t}, \hat{Y}_{t}, \hat{Z}_{t}, \hat{\Gamma}_{t}\right)-f\left(t, \hat{X}_{t}, \Psi_{t}, \hat{Z}_{t}, \hat{\Gamma}_{t}\right)\right] d t .
$$

In the previous step, we proved that $\hat{Y}$ and $\Psi$ are bounded, say by $N$. Since the nonlinearity $f$ is locally bounded, we use the estimate (4.6) to conclude that

$$
\hat{Y}_{\theta}-V\left(\theta, \hat{X}_{\theta}\right) \geq \eta(1-2 \lambda)-\frac{1}{2}\|\beta\| T F_{N} e^{F_{N} T} \lambda^{2} \eta^{2} \geq 0
$$

for all sufficiently small $\lambda$. This is in contradiction to (GDP2). Hence, the proof of the viscosity property is complete.

4.2. The viscosity supersolution property. The proof is almost as in [6]. First we approximate the value function by

$$
V^{m}(s, x):=\inf \left\{y \in \mathbb{R} \mid \exists Z \in \mathcal{A}_{m}^{s, x} \text { so that } Y_{T}^{s, x, y, Z} \geq g\left(X_{T}^{s, x}\right) \text {, a.s. }\right\} .
$$

Then, following the proof of (3.3) in section 5, we can prove the following analogue statement of (GDP1) for $V^{m}$. 
(GDP1m) For every $\varepsilon>0$ and stopping time $\theta \in[s, T]$, there exist $Z_{\varepsilon} \in \mathcal{A}_{m}^{s, x}$ and $y_{\varepsilon} \in\left[V^{m}(s, x), V^{m}(s, x)+\varepsilon\right]$ such that $Y_{\theta}^{s, x, y_{\varepsilon}, Z_{\varepsilon}} \geq V^{m}\left(\theta, X_{\theta}^{s, x}\right)$.

Lemma 4.2. $V_{*}^{m}$ is a viscosity supersolution of (2.4). Consequently, $V_{*}$ is a viscosity supersolution of (2.4).

Proof. This proof of the viscosity property of $V_{*}^{m}$ is exactly the same as the proof of Theorem 4.2 in [6], with one difference. This difference is due to the different assumption (2.2). In [6], instead of (2.2) a uniform $L^{\infty}$ bound was assumed. This change only affects the proof of Lemma 4.4 in [6]. Thus, we follow the proof of Theorem 4.2 in [6] but replace Lemma 4.2 of [6] by Lemma 4.3 below. But for completeness, we will give the proof in detail.

Choose $\left(t_{0}, x_{0}\right) \in[s, T) \times \mathbb{R}^{d}$ and $\varphi \in C^{\infty}\left([s, T) \times \mathbb{R}^{d}\right)$ such that

$$
0=\left(V_{*, s}^{m}-\varphi\right)\left(t_{0}, x_{0}\right)=\min _{(t, x) \in[s, T) \times \mathbb{R}^{d}}\left(V_{*, s}^{m}-\varphi\right)(t, x) .
$$

Let $\left(t_{n}, x_{n}\right)_{n \geq 1}$ be a sequence in $[s, T) \times \mathbb{R}^{d}$ such that $\left(t_{n}, x_{n}\right) \rightarrow\left(t_{0}, x_{0}\right)$ and $V^{m}\left(t_{n}, x_{n}\right)$ $\rightarrow V_{*, s}^{m}\left(t_{0}, x_{0}\right)$. There exist positive numbers $\varepsilon_{n} \rightarrow 0$ such that for $y_{n}=V^{m}\left(t_{n}, x_{n}\right)+$ $\varepsilon_{n}$, there exists $Z^{n} \in \mathcal{A}_{m}^{t_{n}, x_{n}}$ with

$$
Y_{T}^{n} \geq g\left(X_{T}^{n}\right)
$$

where we use the compact notation $\left(X^{n}, Y^{n}\right)=\left(X^{t_{n}, x_{n}}, Y^{t_{n}, x_{n}, y_{n}, Z^{n}}\right)$ and

$$
\begin{aligned}
& Z_{r}^{n}=z_{n}+\int_{t_{n}}^{r} A_{u}^{n} d u+\int_{t_{n}}^{r} \Gamma_{u}^{n} d X_{u}^{n}, \\
& \Gamma_{r}^{n}=\gamma_{n}+\int_{t_{n}}^{r} a_{u}^{n} d u+\int_{t_{n}}^{r} \xi_{u}^{n} d X_{u}^{n}, \quad r \in\left[t_{n}, T\right] .
\end{aligned}
$$

Moreover, $\left|z_{n}\right|,\left|\gamma_{n}\right| \leq m\left(1+\left|x_{n}\right|^{p}\right)$ by assumption (2.1). Hence, by passing to a subsequence, we can assume that $z_{n} \rightarrow z_{0} \in \mathbb{R}^{d}$ and $\gamma_{n} \rightarrow \gamma_{0} \in \mathcal{S}^{d}$. Observe that $\alpha_{n}:=y_{n}-\varphi\left(t_{n}, x_{n}\right) \rightarrow 0$. We choose a decreasing sequence of numbers $\delta_{n} \in\left(0, T-t_{n}\right)$ such that $\delta_{n} \rightarrow 0$ and $\alpha_{n} / \delta_{n} \rightarrow 0$. By (GDP1m),

$$
Y_{t_{n}+\delta_{n}}^{n} \geq V^{m}\left(t_{n}+\delta_{n}, X_{t_{n}+\delta_{n}}^{n}\right),
$$

and therefore,

$$
Y_{t_{n}+\delta_{n}}^{n}-y_{n}+\alpha_{n} \geq \varphi\left(t_{n}+\delta_{n}, X_{t_{n}+\delta_{n}}^{n}\right)-\varphi\left(t_{n}, x_{n}\right),
$$

which, after two applications of Itô's formula, becomes

$$
\begin{aligned}
\alpha_{n} & +\int_{t_{n}}^{t_{n}+\delta_{n}}\left[f\left(r, X_{r}^{n}, Y_{r}^{n}, Z_{r}^{n}, \Gamma_{r}^{n}\right)-\varphi_{t}\left(r, X_{r}^{n}\right)\right] d r \\
& +\left[z_{n}-D \varphi\left(t_{n}, x_{n}\right)\right]^{\prime}\left[X_{t_{n}+\delta_{n}}^{n}-x_{n}\right] \\
& +\int_{t_{n}}^{t_{n}+\delta_{n}}\left(\int_{t_{n}}^{r}\left[A_{u}^{n}-\mathcal{L} D \varphi\left(u, X_{u}^{n}\right)\right] d u\right)^{\prime} \circ d X_{r}^{n} \\
& +\int_{t_{n}}^{t_{n}+\delta_{n}}\left(\int_{t_{n}}^{r}\left[\Gamma_{u}^{n}-D^{2} \varphi\left(u, X_{u}^{n}\right)\right] d X_{u}^{n}\right)^{\prime} \circ d X_{r}^{n} \geq 0 .
\end{aligned}
$$


It is shown in Lemma 4.3 below that the sequence of random vectors

$$
\left(\begin{array}{c}
\delta_{n}^{-1} \int_{t_{n}}^{t_{n}+\delta_{n}}\left[f\left(r, X_{r}^{n}, Y_{r}^{n}, Z_{r}^{n}, \Gamma_{r}^{n}\right)-\varphi_{t}\left(r, X_{r}^{n}\right)\right] d r \\
\delta_{n}^{-1 / 2}\left[X_{t_{n}+\delta_{n}}^{n}-x_{n}\right] \\
\delta_{n}^{-1} \int_{t_{n}}^{t_{n}+\delta_{n}}\left(\int_{t_{n}}^{r}\left[A_{u}^{n}-\mathcal{L} D \varphi\left(u, X_{u}^{n}\right)\right] d u\right)^{\prime} \circ d X_{r}^{n} \\
\delta_{n}^{-1} \int_{t_{n}}^{t_{n}+\delta_{n}}\left(\int_{t_{n}}^{r}\left[\Gamma_{u}^{n}-D^{2} \varphi\left(u, X_{u}^{n}\right)\right] d X_{u}^{n}\right)^{\prime} \circ d X_{r}^{n}
\end{array}\right), n \geq 1
$$

converges in distribution to

$$
\left(\begin{array}{c}
f\left(t_{0}, x_{0}, \varphi\left(t_{0}, x_{0}\right), z_{0}, \gamma_{0}\right)-\varphi_{t}\left(t_{0}, x_{0}\right) \\
\sigma\left(x_{0}\right) W_{1} \\
0 \\
\frac{1}{2} W_{1}^{\prime} \sigma\left(x_{0}\right)^{\prime}\left[\gamma_{0}-D^{2} \varphi\left(t_{0}, x_{0}\right)\right] \sigma\left(x_{0}\right) W_{1}
\end{array}\right)
$$

Set $\eta_{n}=\left|z_{n}-D \varphi\left(t_{n}, x_{n}\right)\right|$, and assume $\delta_{n}^{-1 / 2} \eta_{n} \rightarrow \infty$ along a subsequence. Then, along a further subsequence, $\eta_{n}^{-1}\left(z_{n}-D \varphi\left(t_{n}, x_{n}\right)\right)$ converges to some $\eta_{0} \in \mathbb{R}^{d}$ with

$$
\left|\eta_{0}\right|=1 \text {. }
$$

Multiplying inequality (4.7) with $\delta_{n}^{-1 / 2} \eta_{n}^{-1}$ and passing to the limit yields

$$
\eta_{0}^{\prime} \sigma\left(x_{0}\right) W_{1} \geq 0
$$

which, since $\sigma\left(x_{0}\right)$ is invertible, contradicts (4.10). Hence, the sequence $\left(\delta_{n}^{-1 / 2} \eta_{n}\right)$ has to be bounded, and therefore, possibly after passing to a subsequence,

$$
\delta_{n}^{-1 / 2}\left[z_{n}-D \varphi\left(t_{n}, x_{n}\right)\right] \quad \text { converges to some } \xi_{0} \in \mathbb{R}^{d} .
$$

It follows that $z_{0}=D \varphi\left(t_{0}, x_{0}\right)$. Moreover, we can divide inequality (4.7) by $\delta_{n}$ and pass to the limit to get

$$
\begin{aligned}
& f\left(t_{0}, x_{0}, \varphi\left(t_{0}, x_{0}\right), D \varphi\left(t_{0}, x_{0}\right), \gamma_{0}\right)-\varphi_{t}\left(t_{0}, x_{0}\right) \\
+ & \xi_{0}^{\prime} \sigma\left(x_{0}\right) W_{1}+\frac{1}{2} W_{1}^{\prime} \sigma\left(x_{0}\right)^{\prime}\left[\gamma_{0}-D^{2} \varphi\left(t_{0}, x_{0}\right)\right] \sigma\left(x_{0}\right) W_{1} \geq 0 .
\end{aligned}
$$

Since the support of the random vector $W_{1}$ is $\mathbb{R}^{d}$, it follows from (4.11) that

$$
\begin{aligned}
& f\left(t_{0}, x_{0}, \varphi\left(t_{0}, x_{0}\right), D \varphi\left(t_{0}, x_{0}\right), \gamma_{0}\right)-\varphi_{t}\left(t_{0}, x_{0}\right) \\
& \quad+\xi_{0}^{\prime} \sigma\left(x_{0}\right) w+\frac{1}{2} w^{\prime} \sigma\left(x_{0}\right)^{\prime}\left[\gamma_{0}-D^{2} \varphi\left(t_{0}, x_{0}\right)\right] \sigma\left(x_{0}\right) w \geq 0
\end{aligned}
$$

for all $w \in \mathbb{R}^{d}$. This shows that

$$
f\left(t_{0}, x_{0}, \varphi\left(t_{0}, x_{0}\right), D \varphi\left(t_{0}, x_{0}\right), \gamma_{0}\right)-\varphi_{t}\left(t_{0}, x_{0}\right) \geq 0 \quad \text { and } \quad \beta:=\gamma_{0}-D^{2} \varphi\left(t_{0}, x_{0}\right) \geq 0,
$$

and therefore,

$$
-\varphi_{t}\left(t_{0}, x_{0}\right)+\sup _{\beta \in \mathcal{S}_{+}^{d}} f\left(t_{0}, x_{0}, \varphi\left(t_{0}, x_{0}\right), D \varphi\left(t_{0}, x_{0}\right), D^{2} \varphi\left(t_{0}, x_{0}\right)+\beta\right) \geq 0 .
$$

This proves that $V^{m}$ is a viscosity supersolution. 
Since by definition

$$
V=\inf _{m} V^{m}
$$

by the classical stability property of viscosity solutions, $V_{*}$ is also a viscosity supersolution of the dynamic programming equation (2.4). The detailed argument is identical to the proof of Corollary 5.5 in [4].

LEMma 4.3. The sequence of random vectors (4.8), on a subsequence, converges in distribution to (4.9).

Proof. Define a stopping time by

$$
\tau_{n}:=\inf \left\{r \geq t_{n}: X_{r}^{n} \notin B_{1}\left(x_{0}\right)\right\} \wedge\left(t_{n}+\delta_{n}\right),
$$

where $B_{1}\left(x_{0}\right)$ denotes the open unit ball in $\mathbb{R}^{d}$ around $x_{0}$. It follows from the fact that $x_{n} \rightarrow x_{0}$ that

$$
P\left[\tau_{n}<t_{n}+\delta_{n}\right] \rightarrow 0
$$

Thus in (4.8) we may replace the upper limits of the integrations by $\tau_{n}$ instead of $t_{n}+\delta_{n}$.

Therefore, in the interval $\left[t_{n}, \tau_{n}\right]$ the process $X^{n}$ is bounded. Moreover, in view of (2.2) so are $Z^{n}, \Gamma^{n}$, and $\xi^{n}$.

Step 1. The convergence of the second component of (4.8) is straightforward and the details are exactly as in Lemma 4.4 of [6].

Step 2. Let $B$ be as in (2.1). To analyze the other components, set

$$
A^{n, *}:=\sup _{u \in\left[t_{n}, T\right]} \frac{\left|A_{u}^{n}\right|}{1+\left|X_{u}^{n}\right|^{B}}
$$

so that, by $(2.2)$,

$$
\left\|A^{n, *}\right\|_{L^{(1 / m)}(\Omega, \mathbb{P})} \leq m .
$$

Moreover, since on the interval $\left[t_{n}, \tau_{n}\right], X^{n}$ is uniformly bounded by a deterministic constant $C\left(x_{0}\right)$ depending only on $x_{0}$,

$$
\left|A_{u}^{n}\right| \leq C\left(x_{0}\right) A^{n, *} \leq C\left(x_{0}\right) m \quad \forall u \in\left[t_{n}, \tau_{n}\right] .
$$

(Here and below, the constant $C\left(x_{0}\right)$ may change from line to line.) We define $a^{n, *}$ similarly. Then it also satisfies the above bounds as well. In view of $(2.2), a^{n, *}$ also satisfies (4.12). Moreover, using (2.1), we conclude that $\xi_{u}^{n}$ is uniformly bounded by $m$.

Step 3. Recall that $d \Gamma_{u}^{n}=a_{u}^{n} d u+\xi_{u}^{n} d X_{u}^{n}, \Gamma_{t_{n}}^{n}=\gamma_{n}$. Using the notation and the estimates of the previous step, we directly calculate that

$$
\begin{aligned}
\sup _{t \in\left[t_{n}, \tau_{n}\right]}\left|\Gamma_{t}^{n}-\gamma_{n}\right| & \leq C\left(x_{0}\right) \delta_{n} a^{n, *}+\left|\int_{t_{n}}^{\tau_{n}} \xi_{u}^{n} \cdot \mu^{n} d u\right|+\left|\int_{t_{n}}^{\tau_{n}} \xi_{u}^{n} \sigma\left(X_{u}^{n}\right) d W_{u}\right| \\
& :=I_{1}^{n}+I_{2}^{n}+I_{3}^{n} .
\end{aligned}
$$

Then

$$
E\left[\left(I_{3}^{n}\right)^{2}\right] \leq E\left(\int_{t_{n}}^{\tau_{n}}\left|\xi_{u}^{n}\right|^{2}|\sigma|^{2} d u\right) \leq \delta_{n} m^{2} C\left(x_{0}\right)^{2}
$$


Hence, $I_{3}^{n}$ converges to zero in $L^{2}$. Therefore, it also converges almost surely on a subsequence. We prove the convergence of $I_{2}^{n}$ using similar estimates. Since $a^{n, *}$ satisfies (4.12),

$$
E\left[\left(I_{1}^{n}\right)^{(1 / m)}\right] \leq\left(C\left(x_{0}\right) \delta_{n}\right)^{(1 / m)} E\left[\left|a^{n, *}\right|^{(1 / m)}\right] \leq\left(C\left(x_{0}\right) \delta_{n}\right)^{(1 / m)} m .
$$

Therefore, $I_{1}^{n}$ converges to zero in $L^{(1 / m)}$ and consequently almost surely on a subsequence.

Hence, on a subsequence, $\Gamma_{t}^{n}$ is uniformly continuous. This together with standard techniques used in Lemma 4.4 of [6] proves the convergence of the first component of (4.8).

Step 4 . By integration by parts,

$$
\int_{t_{n}}^{\tau_{n}} \int_{t_{n}}^{t} A_{u}^{n} d u d X_{t}^{n}=\left(X_{\tau_{n}}^{n}-X_{t_{n}}^{n}\right) \int_{t_{n}}^{\tau_{n}} A_{u}^{n} d u-\int_{t_{n}}^{\tau_{n}}\left(X_{u}^{n}-X_{t_{n}}^{n}\right) A_{u}^{n} d u .
$$

Therefore,

$$
\left|\frac{1}{\delta_{n}} \int_{t_{n}}^{\tau_{n}} \int_{t_{n}}^{t} A_{u}^{n} d u d X_{t}^{n}\right| \leq C\left(x_{0}\right) \sup _{t \in\left[t_{n}, \tau_{n}\right]}\left|X_{t}^{n}-X_{t_{n}}^{n}\right| A^{n, *} .
$$

Also $X^{n}$ is uniformly continuous and $A^{n, *}$ satisfies (4.12). Hence, we can show that the above terms, on a subsequence, almost surely converge to zero. This implies the convergence of the third term.

Step 5 . To prove the convergence of the final term it suffices to show that

$$
J^{n}:=\frac{1}{\delta_{n}} \int_{t_{n}}^{\tau_{n}} \int_{t_{n}}^{t}\left[\Gamma_{u}^{n}-\gamma_{n}\right] d X_{u}^{n} \circ d X_{t}^{n}
$$

converges to zero. Indeed, since $\gamma_{n} \rightarrow \gamma_{0}$, this convergence together with the standard arguments of Lemma 4.4 of [6] yields the convergence of the fourth component.

Since on $\left[t_{n}, \tau_{n}\right] X^{n}$ is bounded, on this interval $\left|\sigma\left(X_{t}^{n}\right)\right| \leq C(x)$. Using this bound, we calculate that

$$
\begin{aligned}
E\left[\left(J^{n}\right)^{2}\right] & \leq \frac{C\left(x_{0}\right)^{4}}{\delta_{n}^{2}} \int_{t_{n}}^{t_{n}+\delta_{n}} \int_{t_{n}}^{t} E\left[\mathbf{1}_{\left[t_{n}, \tau_{n}\right]}\left|\Gamma_{u}^{n}-\gamma_{n}\right|^{2}\right] d u d t \\
& \leq C\left(x_{0}\right)^{4} E\left[\sup _{t \in\left[t_{n}, \tau_{n}\right]}\left|\Gamma_{u}^{n}-\gamma_{n}\right|^{2}\right]=: C\left(x_{0}\right)^{4} E\left[\left(e^{n}\right)^{2}\right] .
\end{aligned}
$$

In step 3, we proved the almost sure convergence of $e^{n}$ to zero. Moreover, by (2.1), $\left|e^{n}\right| \leq m$. Therefore, by dominated convergence, we conclude that $J^{n}$ converges to zero in $L^{2}$, and thus almost everywhere on a subsequence.

5. Geometric dynamic programming. This section is dedicated to the proof of Theorem 3.1 .

The main difficulty is related to the continuity of the optimal processes $Z^{*}, \Gamma^{*}$. Indeed, for a stopping time $\theta$ and control $Z \in \mathcal{A}^{t, x}$, set $(\xi, \zeta):=\left(X_{\theta}^{t, x}, Y_{\theta}^{t, x, y, Z}\right)$. Then there exists a control process $\hat{Z} \in \mathcal{A}^{\theta, \xi}$ such that $Y_{T}^{\theta, \xi, \zeta, \hat{Z}} \geq g\left(X_{T}^{t, x}\right)$ (technical measurability issues are resolved in [18]). To prove the dynamic programming, we need to extend the control process to $[t, T]$ by the natural concatenation $\hat{Z}:=Z \mathbf{1}_{[t, \theta)}+$ $\hat{Z} \mathbf{1}_{[\theta, T]}$. However, this construction might lead to a discontinuous control process at $\theta$. 
Therefore, $\hat{Z}$ is not guaranteed to be in $\mathcal{A}^{t, x}$. Similar difficulty exists for the $\Gamma$ process as well. We will resolve this difficulty by modifying the concatenation properly.

We start our analysis by recalling the dynamic programming principle proved by the authors in [18]. Recall that, for $s \in[0, T], x, z \in \mathbb{R}^{d}, \gamma \in \mathcal{S}^{d}$, the control set $\mathcal{A}^{s, x, z, \gamma}$ is defined in Remark 2.1. Set

$$
\mathcal{V}(t):=\left\{(x, y, z, \gamma) \mid \exists Z \in \mathcal{A}^{t, x, z, \gamma} \text { s.t. } Y_{T}^{t, x, y, Z} \geq g\left(X_{T}^{t, x}\right) \text { a.s. }\right\} .
$$

In the above definition, the dependence on the initial conditions $z, \gamma$ is given implicitly through the processes $Z$ and $\Gamma$. Indeed, we suppressed this dependence of $Z, \Gamma$ on the initial data and the further dependencies on the control processes $A, a, \xi$. Moreover, implicitly the processes $Z$ and $\Gamma$ are assumed to be continuous in time. Therefore, to apply the general result proved in [18], we have to include the initial conditions $z$ and $\gamma$ in the reachability set $\mathcal{V}(t)$ defined above. Then, by Theorem 3.1 in [18],

$$
\mathcal{V}(t)=\left\{(x, y, z, \gamma) \mid \exists Z \in \mathcal{A}^{t, x, z, \gamma} \text { s.t. }\left(X_{\theta}^{t, x}, Y_{\theta}^{t, x, y, Z}, Z_{\theta}, \Gamma_{\theta}\right) \in V(\theta) \text { a.s. }\right\}
$$

for any stopping time $\theta \in[t, T]$. Set

$$
\hat{V}(t, x, z, \gamma):=\inf \{y \mid(x, y, z, \gamma) \in \mathcal{V}(t)\} .
$$

Then the dynamic programming principle satisfied by $\mathcal{V}$ translates into an analogous relation for $\hat{V}$. Indeed,

$$
\begin{aligned}
& \hat{V}(t, x, z, \gamma)=\inf \left\{y \mid \exists Z \in \mathcal{A}^{t, x, z, \gamma} \text { s.t. } Y_{T}^{t, x, y, Z} \geq g\left(X_{T}^{t, x}\right) \text { a.s. }\right\} \\
& \quad=\inf \left\{y \mid \exists Z \in \mathcal{A}^{t, x, z, \gamma} \text { s.t. } Y_{\theta}^{t, x, y, Z} \geq \hat{V}\left(\theta, X_{\theta}^{t, x}, Y_{\theta}^{t, x, y, Z}, Z_{\theta}, \Gamma_{\theta}\right) \text { a.s. }\right\} .
\end{aligned}
$$

Therefore to prove Theorem 3.1 or equivalently (3.1), it suffices to show that $\hat{V}(t, x, z, \gamma)$ is independent of $z$ and $\xi$. This is exactly what we will prove in the remainder of this section.

TheOREM 5.1. Suppose $\left(x_{0}, y_{0}, z_{0}, \gamma_{0}\right) \in \mathcal{V}\left(t_{0}\right)$. Then $\left(x_{0}, y, z, \gamma\right) \in \mathcal{V}\left(t_{0}\right)$ for any $y>y_{0}, z \in \mathbb{R}^{d}, \gamma \in \mathcal{S}^{d}$. In particular, $\hat{V}(t, x, z, \gamma)=V(t, x)$ and $(3.1)$ holds.

The proof of this result will be completed in several lemmas. Let $c_{0}$ be as in (A3).

Lemma 5.1. Let $\left(x_{0}, y_{0}, z_{0}, \gamma_{0}\right) \in \mathcal{V}\left(t_{0}\right)$. Then, for every $y>y_{0}$, there exists $Z \in \mathcal{A}^{t_{0}, x_{0}, z_{0}, \gamma_{0}}$ so that almost surely

$$
Y_{t}^{t_{0}, x_{0}, y, Z} \geq Y_{t}^{t_{0}, x_{0}, y_{0}, Z}+\left(y-y_{0}\right) e^{-c_{0}\left(t-t_{0}\right)} \quad \forall t \in\left[t_{0}, T\right],
$$

and $Y_{T}^{t_{0}, x_{0}, y_{0}, Z} \geq g\left(X_{T}^{t_{0}, x_{0}}\right)$. In particular, $\left(x_{0}, y, z_{0}, \gamma_{0}\right) \in \mathcal{V}\left(t_{0}\right)$.

Proof. To simplify the notation, set $\mathcal{A}^{0}:=\mathcal{A}^{t_{0}, x_{0}, z_{0}, \gamma_{0}}, X:=X^{t_{0}, x_{0}}$. Since $\left(x_{0}, y_{0}\right.$, $\left.z_{0}, \gamma_{0}\right) \in \mathcal{V}\left(t_{0}\right)$, there exists a control process $Z \in \mathcal{A}^{0}$ so that

$$
Y_{T}^{0}:=Y_{T}^{t_{0}, x_{0}, y_{0}, Z} \geq g\left(X_{T}\right) \text { a.s. }
$$

Set $Y:=Y^{t_{0}, x_{0}, y, Z}$ so that the error process is given by $\delta:=Y-Y^{0}$. Clearly, $\delta_{t_{0}}=y-y_{0}>0$. Let $\tau:=T \wedge \inf \left\{t \geq t_{0}: \delta_{t} \leq 0\right\}$. Then, for all $u \in\left[t_{0}, \tau\right]$, we have

$$
d \delta_{u}=\left[f\left(u, X_{u}, Y_{u}, Z_{u}, \Gamma_{u}\right)-f\left(u, X_{u}, Y_{u}^{0}, Z_{u}, \Gamma_{u}\right)\right] d u \geq-c_{0} \delta_{u} d u,
$$

where the last inequality follows from (A3). We now use Gronwall's inequality to conclude that $\delta_{u} \geq\left(y-y_{0}\right) e^{-c_{0}\left(u-t_{0}\right)}$ for $u \in\left[t_{0}, \tau\right]$. Hence $\tau=T$ and

$$
Y_{t}=Y_{t}^{0}+\delta_{t} \geq Y_{t}^{0}+\left(y-y_{0}\right) e^{-c_{0}\left(t-t_{0}\right)} .
$$


Lemma 5.2. Let $\left(x_{0}, y_{0}, z_{0}, \gamma_{0}\right) \in \mathcal{V}\left(t_{0}\right)$. Then $\left(x_{0}, y, z, \gamma_{0}\right) \in \mathcal{V}\left(t_{0}\right)$ for any $y>y_{0}$ and $z \in \mathbb{R}^{d}$.

Proof. Fix $\left(x_{0}, y_{0}, z_{0}, \gamma_{0}\right) \in \mathcal{V}\left(t_{0}\right), y>y_{0}$ and $z \in \mathbb{R}^{d}$. We need to construct $Z^{*} \in \mathcal{A}^{t_{0}, x_{0}, z, \gamma_{0}}$ so that the corresponding $Y^{*}=Y^{t_{0}, x_{0}, y, Z^{*}}$ process is superreplicating. Set $X:=X^{t_{0}, x_{0}}$.

Step 1. The building block for $Z^{*}$ is as follows. As in the previous lemma, let $Z \in \mathcal{A}^{0}=\mathcal{A}^{t_{0}, x_{0}, z_{0}, \gamma_{0}}$ be chosen so that $Y^{0}:=Y^{t_{0}, x_{0}, y_{0}, Z}$ is superreplicating. Fix a constant $M>2 /\left(T-t_{0}\right)$ and define a control process $\hat{Z}$ by

$$
\hat{Z}_{u}:=Z_{u}+\left[\left(1-M\left(u-t_{0}\right)\right)\left(z-z_{0}\right)\right] \mathbf{1}_{\left[t_{0}, t_{0}+\frac{1}{M}\right)} .
$$

Note that since $Z \in \mathcal{A}^{t_{0}, x_{0}, z_{0}, \gamma_{0}}, Z_{t_{0}}=z_{0}$. Let $\hat{A}, \hat{\Gamma}$ be defined through $\hat{Z}$. Then this control process has the following properties: $\hat{Z}_{t_{0}}=z, \hat{\Gamma}=\Gamma$ everywhere, $\hat{Z}=Z$ on $\left[t_{0}+1 / M, T\right]$, and

$$
\hat{A}_{u}:=A_{u}+M \mathbf{1}_{\left[t_{0}, t_{0}+\frac{1}{M}\right)} .
$$

In particular, $\hat{Z} \in \mathcal{A}^{t_{0}, x_{0}, z, \gamma_{0}}$.

As in the proof of the preceding lemma, let $Y^{0}=Y^{t_{0}, x_{0}, y_{0}, Z}$. Further set

$$
\bar{Y}_{u}:=Y_{u}^{0}+\frac{1}{2}\left(y-y_{0}\right) e^{-c_{0}\left(t-t_{0}\right)},
$$

$\hat{Y}=Y^{t_{0}, x_{0}, y, \hat{Z}}$, and $R_{0}=1$. Define a stopping time $\tau$ by

$$
\tau:=\left(t_{0}+\frac{1}{M}\right) \wedge \inf \left\{u \geq t_{0}: \hat{Y}_{u} \leq \bar{Y}_{u}\right\} \wedge \inf \left\{u \geq t_{0}:\left|X_{u}-X_{t_{0}}\right| \geq R_{0}\right\},
$$

since $\hat{Y}_{t_{0}}=y>\bar{Y}_{t_{0}}=y_{0}+\left(y-y_{0}\right) / 2, \tau t_{0}$.

The main property of this construction is as follows. If $\tau=t_{0}+(1 / M)$, then $\hat{Y}_{t_{0}+(1 / M)}=y>\bar{Y}_{t_{0}+(1 / M)}$. Also recall that $\hat{Z}=Z$ on $\left[t_{0}+(1 / M), T\right]$. Hence, when $\tau=t_{0}+(1 / M)$

$$
\begin{aligned}
\hat{Y}_{T} & =Y_{T}^{t_{0}, x_{0}, y, \hat{Z}}=Y_{T}^{\tau, X_{\tau}, \hat{Y}_{\tau}, Z} \\
& \geq Y_{T}^{\tau, X_{\tau}, \bar{Y}_{\tau}, Z} Y_{T}^{\tau, X_{\tau}, Y_{\tau}, Z} \\
& \geq g\left(X_{T}\right) .
\end{aligned}
$$

Since $\hat{Z} \in \mathcal{A}^{t_{0}, x_{0}, z, \gamma_{0}}$, the above implies that $\left(x_{0}, y, z, \gamma_{0}\right) \in \mathcal{V}\left(t_{0}\right)$.

Step 2 . The probability of the event $\left\{\tau<t_{0}+1 / M\right\}$ depends on several quantities. However, we will be mainly interested in its dependence on $M$. So we define

$$
\Psi(M):=\Psi\left(M: t_{0}, x_{0}, y, y_{0}, z, R_{0}, Z\right)=\mathbb{P}\left(\tau<t_{0}+\frac{1}{M}\right) .
$$

In Lemma 5.3, below, we will prove that for any $\lambda<1$,

$$
\lim _{M \rightarrow \infty} M^{\lambda} \Psi(M)=0 .
$$

Step 3. Let $\hat{Z}$ be as in the preceding step. Then $Z \in \mathcal{A}^{t_{0}, x_{0}, z_{0}, \gamma_{0}}$ and $\hat{Z} \in$ $\mathcal{A}^{t_{0}, x_{0}, z, \gamma_{0}}$. Moreover, there exists $m_{0}$ so that $Z \in \mathcal{A}_{m_{0}}^{t_{0}, x_{0}, z_{0}, \gamma_{0}}$ and $\hat{Z} \in \mathcal{A}_{m_{0}}^{t_{0}, x_{0}, z, \gamma_{0}}$. 
Step 4. Choose $M_{1}>1 /\left(T-t_{0}\right)$ so that

$$
M_{1}^{\left(1 / m_{0}\right)} \Psi\left(M_{1}\right) \leq \frac{1}{2}
$$

Let $\hat{Z}, \tau$ be as in Step 1 with $M_{1}$. On the set $\left\{\tau=t_{0}+\left(1 / M_{1}\right)\right\}$, we define $Z^{*}=\hat{Z}$.

Step 5. On $\left\{\tau<t_{0}+\left(1 / M_{1}\right)\right\}$, set

$$
\tau^{1}:=\tau, \quad \hat{Z}^{1}:=\hat{Z}, \quad \bar{Y}^{1}:=\bar{Y}, \quad \hat{Y}^{1}:=\hat{Y} .
$$

Then we reiterate the procedure of Step 1 starting at time $\tau^{1}$. Indeed, set $R_{1}=1 / 2$ and choose $M_{2} \geq M_{1}$ as an $\mathcal{F}_{\tau_{1}}$-measurable random variable so that

$$
M_{2}^{\left(1 / m_{0}\right)} \Psi\left(M_{2}: \tau_{1}, X_{\tau_{1}}, \bar{Y}_{\tau_{1}}^{1}, Y_{\tau_{1}}^{0}, \hat{Z}_{\tau_{1}}^{1}, R_{1}, Z\right) \leq \frac{1}{4}
$$

Set

$$
\begin{gathered}
\hat{Z}_{u}^{2}:= \begin{cases}\hat{Z}_{u}^{1} & \text { on } u \in\left[t_{0}, \tau_{1}\right], \\
{\left[Z_{u}+\left(1-M_{2}\left(u-\tau_{1}\right)\right)\left(\hat{Z}_{\tau_{1}}^{1}-Z_{\tau_{1}}\right)\right]} & \text { on } u \in\left[\tau_{1}, \tau_{1}+\left(1 / M_{2}\right)\right], \\
Z_{u} & \text { on } u \in\left[\tau_{1}+\left(1 / M_{2}\right), T\right],\end{cases} \\
\hat{Y}^{2}:=Y^{\tau_{1}, X_{\tau_{1}}, \hat{Y}_{\tau_{1}}^{1}, \hat{Z}^{2}}, \quad \bar{Y}_{u}^{2}:=Y_{u}^{0}+\frac{1}{4}\left(y-y_{0}\right) e^{-c_{0}\left(u-t_{0}\right)} .
\end{gathered}
$$

Finally, define the stopping time $\tau_{2}$ by

$$
\tau_{2}:=\left(\tau_{1}+\frac{1}{M_{2}}\right) \wedge \inf \left\{u \geq \tau_{1}: \hat{Y}_{u}^{2} \leq \bar{Y}_{u}^{2}\right\} \wedge \inf \left\{u \geq \tau_{1}:\left|X_{u}-X_{\tau_{1}}\right| \geq R_{1}\right\} .
$$

Step 6. On $\left\{\tau_{2}=\tau_{1}+\left(1 / M_{2}\right)\right\}$, we define $Z^{*}=\hat{Z}^{2}$ and stop the procedure. On the complement of this set, we iterate this procedure to obtain a sequence

$$
\left\{M_{k}, \tau_{k}, \hat{Z}^{k}, \hat{Y}^{k}, \bar{Y}^{k}\right\}_{k=1,2, \ldots, N}
$$

so that $R_{k}=2^{-k}, M_{k+1} \geq M_{k}$,

$$
\begin{aligned}
M_{k+1}^{\left(1 / m_{0}\right)} \Psi & \left(M_{k+1}: \tau_{k}, X_{\tau_{k}}, \bar{Y}_{\tau_{k}}^{k}, Y_{\tau_{k}}^{0}, \hat{Z}_{\tau_{k}}^{k}, R_{k}, Z\right) \\
& =M_{k+1}^{\left(1 / m_{0}\right)} \mathbb{P}\left(\tau_{k+1}<\tau_{k}+\frac{1}{M_{k+1}}\right) \leq \frac{1}{2^{k+1}} .
\end{aligned}
$$

Then define $\hat{Z}^{k+1}$ as before with $M_{k+1}$. Also, set

$$
\begin{aligned}
\hat{Y}^{k+1}:=Y^{\tau_{k}, X_{\tau_{k}}, \hat{Y}_{\tau_{k}}^{k}, Z^{k+1},} & \bar{Y}^{k+1}:=Y^{0}+R_{k+1}\left(y-y_{0}\right) e^{-c_{0}\left(u-t_{0}\right)}, \\
\tau_{k+1}:=\left(\tau_{k}+\frac{1}{M_{k+1}}\right) & \wedge \inf \left\{u \geq \tau_{k}: \hat{Y}_{u}^{k+1} \leq \bar{Y}_{u}^{k+1}\right\} \\
& \wedge \inf \left\{u \geq \tau_{k}:\left|X_{u}-X_{\tau_{k}}\right| \geq R_{k}\right\} .
\end{aligned}
$$


The random integer $N$ is the step at which this procedure stops, i.e., $\left\{\tau_{N}=\tau_{N-1}+\right.$ $\left.\left(1 / M_{N}\right)\right\}$. As before, on this set we define $Z^{*}:=\hat{Z}^{N}$. Then $Y^{*}=Y^{t_{0}, x_{0}, y, Z^{*}}=\hat{Y}^{N}$. By construction, $Y_{T}^{*} \geq g\left(X_{T}\right)$ a.s. Hence, we may conclude that $\left(x_{0}, y, z, \gamma_{0}\right) \in \mathcal{V}\left(t_{0}\right)$, provided that $N<\infty$ a.s. and that $Z^{*} \in \mathcal{A}^{t_{0}, x_{0}, y, z}$. These will be shown in the next two steps.

Step 7. In view of (5.1),

$$
\sum_{k=1}^{\infty} \mathbb{P}\left(\tau_{k}<\tau_{k-1}+\frac{1}{M_{k}}\right) \leq \sum_{k=1}^{\infty} \frac{1}{2^{k}}<\infty .
$$

Therefore, by the Borel-Cantelli lemma, $\mathbb{P}(N<\infty)$ is equal to one.

Step 8. Recall that $d Z_{t}=A_{t} d t+\Gamma_{t} d W_{t}$ and $Z \in \mathcal{A}_{m_{0}}^{t_{0}, x_{0}, z_{0}, \gamma_{0}}$. Hence,

$$
\|A\|_{t_{0}, x_{0}}^{B,\left(1 / m_{0}\right)} \leq m_{0}
$$

Let $\Gamma^{*}$ and $A^{*}$ be the control processes corresponding to $Z^{*}$. Then $\Gamma^{*}=\Gamma$, in particular $\Gamma_{t_{0}}^{*}=\gamma_{0}$. Moreover, by construction $Z_{t_{0}}^{*}=z$ and $|\hat{Z}-Z| \leq\left|z-z_{0}\right|$. Hence, to verify that $Z^{*} \in \mathcal{A}^{t_{0}, x_{0}, z, \gamma_{0}}$, we need to show that for some $m$,

$$
\left\|A^{*}\right\|_{t_{0}, x_{0}}^{B,(1 / m)} \leq m
$$

By definition,

$$
A_{u}^{*}=A_{u}+\sum_{k} M_{k} \mathbf{1}_{\left[\tau_{k-1}, \tau_{k}\right)}(u)
$$

Since $M_{k}$ is nondecreasing in $k$, the maximum over $u$ of the summation term in the above expression is achieved for the final $k$, i.e., when $N=k$. Hence,

$$
\sup _{t_{0} \leq u \leq T} \frac{\left|A_{u}^{*}\right|}{1+\left|X_{u}\right|^{B}} \leq \sup _{t_{0} \leq u \leq T} \frac{\left|A_{u}\right|}{1+\left|X_{u}\right|^{B}}+\sum_{k} M_{k} \mathbf{1}_{\left\{N=\tau_{k}\right\}} .
$$

Since $\left\{N=\tau_{k}\right\} \subset\left\{N \geq \tau_{k}\right\} \subset\left\{\tau_{k}<\tau_{k-1}+1 / M_{k}\right\}$,

$$
\begin{aligned}
\left\|A^{*}\right\|_{t_{0}, x_{0}}^{B,\left(1 / m_{0}\right)} \leq & \|A\|_{t_{0}, x_{0}}^{B,\left(1 / m_{0}\right)} \\
& +\sum_{k}\left(M_{k}\right)^{\left(1 / m_{0}\right)} \mathbb{P}\left(\tau_{k}<\tau_{k-1}+\frac{1}{M_{k}}\right) \leq m_{0}+1 .
\end{aligned}
$$

Remark 5.1. In the proof of the above lemma, the dynamics of the processes are not used. In fact, the above lemma is a recursive procedure to construct the control process. The technical input for this procedure is given through two technical results, Lemma 5.1, proved prior to the lemma, and Lemma 5.3, proved below. However, note that Lemma 5.1 is just a simple consequence of the monotonicity assumption (A3). Hence, for any other situation with an $f$ satisfying (A3) to obtain the conclusions of Lemma 5.2, it suffices to prove the technical estimate proved in Lemma 5.3 below.

Remark 5.2. In the above proof, the construction of the exit times shows that for $u \in\left[t_{0}, \tau_{k+1}\right]$,

$$
\left|X_{u}-x_{0}\right| \leq\left|X_{u}-X_{\tau_{k}}\right|+\left|X_{\tau_{k}}-X_{\tau_{k-1}}\right|+\cdots+\left|X_{\tau_{1}}-x_{0}\right| \leq R_{k}+\cdots+R_{0} \leq 2 .
$$

Hence, for $u \in\left[t_{0}, \tau_{N}\right]$, the process $X$ is uniformly bounded. This is the only reason to introduce the exit times in the definition of $\tau_{k}$. Indeed, this property will be used in the proof of Lemma 5.4 below. 
The following technical estimate is used in the proof of Lemma 5.2.

Lemma 5.3. Let $\Psi$ be as in Step 2 in the proof of Lemma 5.2. Then, for every $\lambda<1$,

$$
\lim _{M \rightarrow \infty} M^{\lambda} \Psi\left(M: t_{0}, x_{0}, y, y_{0}, z, R, Z\right)=0 .
$$

Proof. Recall that $\tau:=\left(t_{0}+(1 / M)\right) \wedge \tau_{1} \wedge \tau_{2}$, where

$$
\tau_{1}:=\inf \left\{u \geq t_{0} \hat{Y}_{u} \leq \bar{Y}_{u}\right\}, \quad \inf \left\{u \geq t_{0}\left|X_{u}-x_{0}\right| \leq R\right\} .
$$

Clearly,

$$
\mathbb{P}\left[\tau_{2} \geq t_{0}+\frac{1}{M}\right] \leq \frac{C(R)}{M}
$$

for some $C(R)$.

Using the notation of the previous proof, set $\delta_{u}:=\hat{Y}_{u}-\bar{Y}_{u}$. By definition, $\delta_{t_{0}}=\left(y-y_{0}\right) / 2:=\eta$ and $\delta_{u} \geq 0$ on $\left[t_{0}, \tau\right]$. Also, by the definition of the process $\hat{Z}$,

$$
\hat{Z}_{u}-Z_{u}=\left(z-z_{0}\right)\left[1-M\left(u-t_{0}\right)\right] \text { for } u \in\left[t_{0}, \tau\right],
$$

and therefore

$$
\left|\hat{Z}_{u}-Z_{u}\right| \leq\left|z-z_{0}\right| \text { for } u \in\left[t_{0}, \tau\right] .
$$

Recall that $\hat{Z}$ and $Z$ have the same diffusion coefficient (i.e., the same $\Gamma$ ). Also $\bar{Y}_{u}=Y_{u}^{0}+\eta e^{-c_{0}\left(u-t_{0}\right)}$. Now, set

$$
\hat{\delta}_{u}:=\delta_{u} e^{c_{0}\left(u-t_{0}\right)}=e^{c_{0}\left(u-t_{0}\right)}\left[\hat{Y}_{u}-Y_{u}^{0}\right]-\eta .
$$

Then we directly compute that

$$
\begin{aligned}
\hat{\delta}_{u}= & \eta+\int_{t_{0}}^{u} e^{c_{0}\left(r-t_{0}\right)}\left[d\left(\hat{Y}_{u}-Y_{u}^{0}\right)+c_{0}\left(\hat{Y}_{u}-Y_{u}^{0}\right)\right] \\
= & \eta+\int_{t_{0}}^{u} e^{c_{0}\left(r-t_{0}\right)}\left[\left(\hat{Z}_{r}-Z_{r}\right) \circ d X_{r}\right. \\
& \left.+\left(f\left(r, X_{r}, \hat{Y}_{r}, \hat{Z}_{r}, \Gamma_{r}\right)-f\left(r, X_{r}, Y_{r}^{0}, Z_{r}, \Gamma_{r}\right)+c_{0}\left(\hat{Y}_{u}-Y_{u}^{0}\right)\right) d r\right] \\
\geq & \eta\left(z-z_{0}\right) \int_{t_{0}}^{u} e^{c_{0}\left(r-t_{0}\right)}\left[1-M\left(r-t_{0}\right)\right] \cdot d X_{r} \\
& +\int_{t_{0}}^{u} e^{c_{0}\left(r-t_{0}\right)}\left[f\left(r, X_{r}, Y_{r}^{0}, \hat{Z}_{r}, \Gamma_{r}\right)-f\left(r, X_{r}, Y_{r}^{0}, Z_{r}, \Gamma_{r}\right)\right] d r,
\end{aligned}
$$

where we used condition (A3) in the third step. Hence

$$
\hat{\delta}_{u} \geq \eta+\int_{t_{0}}^{u} \phi_{r} d r+\int_{t_{0}}^{u} m_{r} \cdot d W_{r}
$$

where $m_{r}:=e^{c_{0}(r-t)}\left[1-M\left(r-t_{0}\right)\right] \sigma\left(X_{r}\right)\left(z-z_{0}\right)$, and

$$
\begin{aligned}
\phi_{r}:= & e^{c_{0}\left(r-t_{0}\right)}\left[f\left(r, X_{r}, Y_{r}, \hat{Z}_{r}, \Gamma_{r}\right)-f\left(r, X_{r}, Y_{r}, Z_{r}, \Gamma_{r}\right)\right] \\
& +e^{c_{0}\left(r-t_{0}\right)}\left[1-M\left(r-t_{0}\right)\right]\left(z-z_{0}\right) \cdot \mu\left(X_{r}\right) .
\end{aligned}
$$


Moreover, they both satisfy

$$
\left|\phi_{r}\right|+\left|m_{r}\right| \leq C\left(1+\left|X_{r}\right|^{P}\right)
$$

for some $C, P>0$. We now estimate that

$$
\begin{aligned}
\mathbb{P}\left[\tau_{1} \geq t_{0}+\frac{1}{M}\right] & =\mathbb{P}\left[\inf _{t_{0} \leq u \leq t_{0}+\frac{1}{M}} \hat{\delta}_{u} \geq 0\right] \\
& \geq \mathbb{P}\left[\inf _{t_{0} \leq u \leq t_{0}+\frac{1}{M}} \int_{t_{0}}^{u} \phi_{r} d r+\int_{t_{0}}^{u} m_{r} \cdot d W_{r} \geq-\eta\right] \\
& =1-\mathbb{P}\left[\sup _{t_{0} \leq u \leq t_{0}+\frac{1}{M}} \int_{t_{0}}^{u} \phi_{r} d r+\int_{t_{0}}^{u} m_{r} \cdot d W_{r} \geq \eta\right] \\
& \geq 1-\eta^{-2} \mathbb{E}\left[\left(\sup _{t_{0} \leq u \leq t_{0}+\frac{1}{M}} \int_{t_{0}}^{u} \phi_{r} d r+\int_{t_{0}}^{u} m_{r} \cdot d W_{r}\right)^{2}\right]
\end{aligned}
$$

by the Chebyshev inequality. It then follows from the Burkholder-Davis-Gundy inequality that

$$
\begin{aligned}
\mathbb{P}\left[\tau_{1}<t_{0}+\frac{1}{M}\right] & \leq 2 \eta^{-2} \mathbb{E}\left[\left(\int_{t_{0}}^{t_{0}+\frac{1}{M}}\left|\phi_{r}\right| d r\right)^{2}+\left(\sup _{t_{0} \leq u \leq t_{0}+\frac{1}{M}} \int_{t_{0}}^{u} m_{r} \cdot d W_{r}\right)^{2}\right] \\
& \leq C \eta^{-2} E\left[\left(\int_{t_{0}}^{t_{0}+\frac{1}{M}}\left|\phi_{r}\right| d r\right)^{2}+\int_{t_{0}}^{t_{0}+\frac{1}{M}}\left|m_{r}\right|^{2} d r\right] \\
& \leq \frac{C}{M \eta^{2}}\left(1+\mathbb{E}\left[\sup _{r \in\left[t_{0}, T\right]}\left|X_{r}\right|^{2 P}\right]\right)
\end{aligned}
$$

since

$$
\Psi(M) \leq \mathbb{P}\left[\tau_{1}<t_{0}+\frac{1}{M}\right]+\mathbb{P}\left[\tau_{2} \geq t_{0}+\frac{1}{M}\right] \leq \frac{C}{M}
$$

Lemma 5.4. Let $\left(x_{0}, y_{0}, z_{0}, \gamma_{0}\right) \in \mathcal{V}\left(t_{0}\right)$. Then $\left(x_{0}, y, z_{0}, \gamma\right) \in \mathcal{V}\left(t_{0}\right)$ for any $y>y_{0}$ and $\gamma \in \mathcal{S}^{d}$.

Proof. Fix $\left(x_{0}, y_{0}, z_{0}, \gamma_{0}\right) \in \mathcal{V}\left(t_{0}\right), y>y_{0}$ and $\gamma \in \mathcal{S}^{d}$. We need to construct $Z^{*} \in \mathcal{A}^{t_{0}, x_{0}, z_{0}, \gamma}$ so that the corresponding $Y^{*}$ process is superreplicating.

This construction is the same as the one given in the previous proof. In view of Remark 5.1, we need to prove Lemma 5.3 under this new construction. This proof is also very similar to the above proof of Lemma 5.3.

Again let $\hat{\delta}_{u}:=\delta_{u} e^{c_{0}\left(u-t_{0}\right)}$ and $\delta_{u}:=\hat{Y}_{u}-\bar{Y}_{u}$. Also, by the definition of the process $\hat{Z}$, for $u \in\left[t_{0}, \tau\right]$,

$$
\begin{aligned}
\hat{Z}_{u}-Z_{u} & =\int_{t_{0}}^{u}\left[\hat{\Gamma}_{r}-\Gamma_{r}\right] \circ d X_{r}=\left(\gamma-\gamma_{0}\right) \int_{t_{0}}^{u}\left[1-M\left(r-t_{0}\right)\right] \circ d X_{r} \\
& =\left(\gamma-\gamma_{0}\right)\left[\left(X_{u}-x_{0}\right)-M\left(u-t_{0}\right) X_{u}+M \int_{t_{0}}^{u} X_{r} d r\right] .
\end{aligned}
$$


Since $\tau \leq t_{0}+(1 / M)$, for $u \in\left[t_{0}, \tau\right], M\left(u-t_{0}\right) \leq 1$. Moreover, in view of Remark 5.2 ,

$$
\sup _{t_{0} \leq r \leq u}\left|X_{r}^{t_{0}, x_{0}}-x_{0}\right| \leq 2 .
$$

Therefore, there exists a constant $C$,

$$
\left|\hat{Z}_{u}-Z_{u}\right| \leq C\left|\gamma-\gamma_{0}\right|\left[\left|x_{0}\right|+2\right] \text { for } u \in\left[t_{0}, \tau\right] .
$$

Then we directly compute that

$$
\begin{aligned}
\hat{\delta}_{u}= & \eta+\int_{t_{0}}^{u} e^{c_{0}\left(r-t_{0}\right)}\left[d\left(\hat{Y}_{u}-Y_{u}^{0}\right)+c_{0}\left(\hat{Y}_{u}-Y_{u}^{0}\right)\right] \\
= & \eta+\int_{t_{0}}^{u} e^{c_{0}\left(r-t_{0}\right)}\left[\left(\hat{Z}_{r}-Z_{r}\right) \circ d X_{r}\right. \\
& \left.+\left(f\left(r, X_{r}, \hat{Y}_{r}, \hat{Z}_{r}, \hat{\Gamma}_{r}\right)-f\left(r, X_{r}, Y_{r}^{0}, Z_{r}, \Gamma_{r}\right)+c_{0}\left(\hat{Y}_{u}-Y_{u}^{0}\right)\right) d r\right] \\
\geq & \eta+\left(z-z_{0}\right) \int_{t_{0}}^{u} e^{c_{0}\left(r-t_{0}\right)}\left(\hat{Z}_{r}-Z_{r}\right) \circ d X_{r} \\
& +\int_{t_{0}}^{u} e^{c_{0}\left(r-t_{0}\right)}\left[f\left(r, X_{r}, Y_{r}^{0}, \hat{Z}_{r}, \hat{\Gamma}_{r}\right)-f\left(r, X_{r}, Y_{r}^{0}, Z_{r}, \Gamma_{r}\right)\right] d r,
\end{aligned}
$$

where we used condition (A3) in the third step. Hence

$$
\hat{\delta}_{u} \geq \eta+\int_{t_{0}}^{u} \phi_{r} d r+\int_{t_{0}}^{u} m_{r} \cdot d W_{r},
$$

where $m_{r}:=e^{c_{0}(r-t)}\left(\hat{Z}_{r}-Z_{r}\right) \sigma\left(X_{r}\right)$, and

$$
\phi_{r}:=e^{c_{0}\left(r-t_{0}\right)}\left[f\left(r, X_{r}, Y_{r}, \hat{Z}_{r}, \hat{\Gamma}_{r}\right)-f\left(r, X_{r}, Y_{r}, Z_{r}, \Gamma_{r}\right)+\left(\hat{Z}_{r}-Z_{r}\right) \cdot \mu\left(X_{r}\right)\right] .
$$

Moreover, they both satisfy

$$
\left|\phi_{r}\right|+\left|m_{r}\right| \leq C\left(1+\left|X_{r}^{t_{0}, x_{0}}\right|^{P}\right)
$$

for some $C, P>0$. We then proceed exactly as in the proof of Lemma 5.2.

6. Terminal condition. In this section, we first prove Theorem 3.3 under assumption (A5)'. Then we will show that several sets of conditions on the functions $f$ and $g$ imply (A5)'.

Proof of Theorem 3.3. First we observe that under (A5), the inequality $V_{*}(T,$. $g($.$) follows from Proposition 4.5$ in $[6]$.

It remains to prove that $V^{*}(T,) \leq$.$g . Let U^{\varepsilon, x_{0}}(t, x)$ be as in (A5)'. For every $(t, x) \in[0, T) \times \mathbb{R}^{d}$, set $X:=X^{t, x}$. We directly calculate by Itô's formula that for all $t \in\left[t\left(\varepsilon, x_{0}\right), T\right]$,

$$
g\left(X_{T}\right) \leq U^{\varepsilon, x_{0}}\left(X_{T}\right)=U^{\varepsilon, x_{0}}(t, x)+\int_{t}^{T} U_{t}^{\varepsilon, x_{0}} d s+\int_{t}^{T} D U^{\varepsilon, x_{0}} \circ d X_{s} .
$$

Recall that by (A5)', $U^{\varepsilon, x_{0}}$ is a supersolution of (2.4) with $f=\hat{f}$. Hence,

$$
g\left(X_{T}\right) \leq U^{\varepsilon, x_{0}}(t, x)+\int_{t}^{T} f\left(s, X_{s}, U^{\varepsilon, x_{0}}, D U^{\varepsilon, x_{0}}, D^{2} U^{\varepsilon, x_{0}}\right) d s+\int_{t}^{T} D U^{\varepsilon, x_{0}} \circ d X_{s} .
$$


In addition, the process $\left\{Z_{s}:=D U^{\varepsilon, x_{0}}\left(s, X_{s}\right), t \leq s \leq T\right\} \in \mathcal{A}^{t, x}$. Therefore, by the definition of $V(t, x), U^{\varepsilon, x_{0}}(t, x) \geq V(t, x)$ for all $t \in\left[t\left(\varepsilon, x_{0}\right), T\right]$. Since $U^{\varepsilon}$ is continuous up to the final time, $U^{\varepsilon, x_{0}}(T, x) \geq V^{*}(T, x)$. Finally, we send $\varepsilon \downarrow 0$ and use (3.4) at $x_{0}$ to arrive at $g\left(x_{0}\right) \geq V^{*}\left(T, x_{0}\right)$.

We continue by obtaining several sufficient conditions for (A5)'.

Lemma 6.1. Suppose $g$ is bounded and that for every $N$ there is $C_{N}$ so that

$$
f(t, x, y, z, \gamma) \geq-C_{N}[1+|y|] \quad \forall|z|,|\gamma| \leq N .
$$

Then there exists $U^{\varepsilon}$ satisfying (A5)'.

Proof. Let $g^{\varepsilon}$ be a smooth mollification of $g$. Then all derivatives of $g^{\varepsilon}$ are bounded by a constant depending on $\varepsilon$. Set

$$
U^{\varepsilon}(t, x):=g^{\varepsilon}(x)+C(T-t)+\left\|g^{\varepsilon}-g\right\|_{\infty} .
$$

We claim that for sufficiently large $C, U^{\varepsilon}$ is a supersolution of (2.4). Indeed, use the above lower bound (6.1) with $N_{\epsilon}=\sup \left|D U^{\varepsilon}\right|+\left|D^{2} U^{\varepsilon}\right|$. Set $C(\epsilon)=C_{N_{\epsilon}}$. We then directly estimate that

$$
\begin{gathered}
-U_{t}^{\varepsilon}+f\left(t, x, U^{\varepsilon}, D U^{\varepsilon}, D^{2} U^{\varepsilon}\right) \geq C-C(\varepsilon)\left[1+\left|U^{\varepsilon}(t, x)\right|\right] \\
=C-C(\varepsilon)\left[1+\left\|g^{\varepsilon}\right\|_{\infty}+C(T-t)\right] .
\end{gathered}
$$

Then

$$
C-C(\varepsilon)\left[1+\left\|g^{\varepsilon}\right\|_{\infty}+C(T-t)\right]=C[1-C(\varepsilon)(T-t)]-C(\varepsilon)\left[1+\left\|g^{\varepsilon}\right\|_{\infty}\right] \geq 0,
$$

provided that $t \geq T-1 /(2 C(\varepsilon))$ and $C \geq 2 C(\varepsilon)\left[1+\left\|g^{\varepsilon}\right\|_{\infty}\right]$. Also, by definition, $U^{\varepsilon}$ satisfies (3.4).

Lemma 6.2. Suppose $g$ is growing at most linearly, i.e.,

$$
|g(x)| \leq C[1+|x|]
$$

Further suppose that there is $c_{0}$, and that for every $N$ there is $C_{N}$ so that

$$
f(t, x, y, z, \gamma) \geq-\left[c_{0}|y|+C_{N}\right] \quad \forall|z|,|\gamma| \leq N .
$$

Then there exists $U^{\varepsilon}$ satisfying (A5)'.

Proof. Let $Q(x)$ be a smooth function satisfying, for all $x$,

$$
Q(x) \geq Q(0)=0, \quad Q(x) \geq|x| \wedge|x|^{2}, \quad Q(x) \leq 2|x| .
$$

Fix $x_{0}$. Since $g$ is continuous (assumption (A3)), for every $\epsilon>0$ there is $\lambda_{\epsilon}>0$, so that

$$
g(x) \leq g^{\varepsilon}(x):=g\left(x_{0}\right)+\epsilon+Q\left(\lambda_{\epsilon}\left(x-x_{0}\right)\right) \quad \forall x \in \mathbb{R}^{d} .
$$

Moreover, the first two derivatives of $g^{\varepsilon}$ are bounded by a constant, possibly depending on $\varepsilon$ :

$$
c_{\varepsilon}:=\left\|D g^{\varepsilon}\right\|_{\infty}+\left\|D^{2} g^{\varepsilon}\right\|_{\infty}<\infty .
$$

For a large constant $C$ to be determined below, we set

$$
U^{\varepsilon}(t, x)=U^{\varepsilon}\left(t, x ; x_{0}, C\right):=g^{\varepsilon}(x) e^{c_{0}(T-t)}+C(T-t) .
$$


Clearly, $U^{\varepsilon}(T, x) \geq g(x)$ for all $C$. Moreover, for every $\lambda, C$, and $x_{0}$

$$
\lim _{\varepsilon \downarrow 0} U^{\varepsilon}\left(T, x_{0} ; \lambda, C\right)=g\left(x_{0}\right) .
$$

Hence, $U^{\varepsilon}$ satisfies (3.4).

We claim that for sufficiently large $C, U^{\varepsilon}$ is a supersolution of (2.4). Indeed, on $[0, T] \times \mathbb{R}^{d}$

$$
\left\|D U^{\varepsilon}\right\|_{\infty}+\left\|D^{2} U^{\varepsilon}\right\|_{\infty} \leq c_{\varepsilon} e^{c_{0} T} .
$$

Set $C(\varepsilon):=C_{N_{\varepsilon}}$, where $N_{\varepsilon}=c_{\varepsilon} e^{c_{0} T}$, and $C_{N}$ is as in our hypothesis. We directly calculate that

$$
\begin{aligned}
-U_{t}^{\varepsilon}+f\left(t, x, U^{\varepsilon}, D U^{\varepsilon}, D^{2} U^{\varepsilon}\right) & \geq C+c_{0} g^{\varepsilon}(x) e^{c_{0}(T-t)}-\left[c_{0}\left|U^{\varepsilon}(t, x)\right|+C(\varepsilon)\right] \\
& =C-c_{0} C(T-t)-C(\varepsilon) \geq 0,
\end{aligned}
$$

provided that $C \geq 2 C(\varepsilon)$ and $t \geq T-1 /\left(2 c_{0}\right)$.

One may construct supersolutions in the case of polynomial growing terminal data. However, in those cases we need to assume growth in the gradient variable of $f$.

\section{REFERENCES}

[1] P. BANK AND D. BAUM, Hedging and portfolio optimization in financial markets with a large trader, Math. Finance, 14 (2004), pp. 1-18.

[2] B. Bouchard, Stochastic targets with mixed diffusion processes and viscosity solutions, Stochastic Process. Appl., 101 (2002), pp. 273-302.

[3] U. Cetin, H. M. Soner, And N. Touzi, Options hedging for small investors under liquidity costs, Finance Stoch., to appear.

[4] P. Cheridito, H. M. Soner, And N. Touzi, The multi-dimensional super-replication problem under gamma constraints, Ann. Inst. H. Poincaré Anal. Non Linéaire, 22 (2005), pp. 633666.

[5] P. Cheridito, H. M. Soner, And N. Touzi, Small time path behavior of double stochastic integrals and applications to stochastic control, Ann. Appl. Probab., 15 (2005), pp. 24722495.

[6] P. Cheridito, H. M. Soner, N. Touzi, And N. Victoir, Second order BSDE's and fully nonlinear PDE's, Comm. Pure Appl. Math., 60 (2007), pp. 1081-1110.

[7] M. G. Crandall, H. Ishit, And P. L. Lions, User's guide to viscosity solutions of second order partial differential equations, Bull. Amer. Math. Soc., 27 (1992), pp. 1-67.

[8] N. El Karoui, S. Peng, And M. C. Quenez, Backward stochastic differential equations in finance, Math. Finance, 7 (1997), pp. 1-71.

[9] W. H. Fleming And H. M. Soner, Controlled Markov Processes and Viscosity Solutions, Appl. Math. (N.Y.) 25, Springer-Verlag, New York, 1993.

[10] N. Ikeda and S. Watanabe, Stochastic Differential Equations and Diffusion Processes, 2nd ed., North-Holland, Amsterdam, 1989.

[11] I. Karatzas and S. Shreve, Brownian Motion and Stochastic Calculus, 2nd ed., SpringerVerlag, New York, 1991.

[12] S. Levental And A. V. Skorohod, On the possibility of hedging options in the presence of transaction costs, Ann. Appl. Probab., 7 (1997), pp. 410-443.

[13] E. Pardoux and S. Peng, Adapted solution of a backward stochastic differential equation, Systems Control Lett., 14 (1990), pp. 55-61.

[14] S. PENG, Probabilistic interpretation for systems of quasilinear parabolic partial differential equations, Stochastics Stochastics Rep., 37 (1991), pp. 61-74.

[15] N. SANTIER, General stochastic target problem with jump diffusion and application to hedging problem for large investor, Electron. Comm. Probab., 12 (2007), pp. 106-119.

[16] H. M. Soner And N. TouzI, Superreplication under gamma constraints, SIAM J. Control Optim., 39 (2000), pp. 73-96.

[17] H. M. Soner AND N. TouzI, Stochastic target problems, dynamic programming, and viscosity solutions, SIAM J. Control Optim., 41 (2002), pp. 404-424.

[18] H. M. Soner And N. TouzI, Dynamic programming for stochastic target problems and geometric flows, J. Eur. Math. Soc. (JEMS), 8 (2002), pp. 201-236. 